\title{
Estudo FATA: Prevalência de Fibrilhação Auricular e Terapêutica Antitrombótica nos Cuidados de Saúde Primários de um Concelho do Norte de Portugal
}

\author{
FATA Study: Prevalence of Atrial Fibrillation and Antithrombotic Therapy in \\ Primary Health Care in a Northern City of Portugal
}

\section{Eva GOMES ${ }^{1}$, Rui CAMPOS ${ }^{1}$, Renata MORAIS ${ }^{1}$, Marta FERNANDES ${ }^{1}$}

Acta Med Port 2015 Jan-Feb;28(1):35-43

\section{RESUMO}

Introdução: A fibrilhação auricular é a arritmia sustentada mais prevalente. Está provada a eficácia da anticoagulação oral na prevenção do acidente vascular cerebral nestes doentes. Contudo, este parece ser um tratamento subutilizado.

Objectivos: determinar a prevalência de fibrilhação auricular conhecida numa população dos Cuidados de Saúde Primários; identificar as principais comorbilidades, a terapêutica antitrombótica em curso e avaliar a sua adequação segundo as recomendações da European Society of Cardiology.

Material e Métodos: Estudo observacional transversal analítico. População: todos os utentes com idade igual ou superior a 30 anos, inscritos em oito Unidades de Saúde Familiar de Vila Nova de Gaia, com diagnóstico de fibrilhação auricular.

Resultados: A prevalência de fibrilhação auricular foi de 1,29\% $(n=940)$, sendo superior no género masculino $(p=0,01)$ e aumentando com a idade $(p<0,001)$. As comorbilidades mais frequentes foram a hipertensão arterial $(76,4 \%)$, a insuficiência cardíaca $(32,0 \%)$ e a diabetes mellitus $(28,2 \%)$. Um total de $52 \%$ realizava terapêutica anticoagulante, $29 \%$ antiagregantes plaquetários e $4 \%$ ambas as terapêuticas. Dos utentes com baixo risco trombótico, $63,6 \%$ estava a fazer erradamente algum tipo de terapêutica antitrombótica; dos utentes com elevado risco ou doença valvular $56,8 \%$ estava adequadamente sob terapêutica anticoagulante.

Conclusão: A prevalência de fibrilhação auricular bem como a frequência das principais comorbilidades estão de acordo com a maioria dos estudos. Apesar de a maioria dos doentes se encontrar sob anticoagulação oral, apenas $56,8 \%$ dos utentes com fibrilhação auricular fazia terapêutica antitrombótica adequada segundo as recomendações da European Society of Cardiology, verificando-se uma subutilização acentuada deste tratamento.

Palavras-chave: Fibrilhação Auricular; Anticoagulantes; Acidente Vascular Cerebral; Cuidados de Saúde Primários; Portugal.

\section{ABSTRACT}

Introduction: Atrial fibrillation is the most prevalent sustained arrhythmia. The efficacy of oral anticoagulation has been proved in prevention stroke in these patients. However, this seems to be an underutilized treatment.

Objectives: to determine the prevalence of known atrial fibrillation in a Primary Health Care population; to identify major comorbidities, current antithrombotic therapy and evaluate their suitability according to the European Society of Cardiology guidelines.

Material and Methods: Observational cross-sectional analytical study. Population: all patients aged 30 or above, enrolled in eight Family Health Units of Vila Nova de Gaia and diagnosed with atrial fibrillation.

Results: Prevalence of atrial fibrillation was $1.29 \%(n=940)$, being higher in males $(p=0.01)$ and increasing with age $(p<0.001)$. The most common comorbidities were hypertension (76.4\%), heart failure $(32.0 \%)$ and diabetes mellitus $(28.2 \%)$. A total of $52 \%$ was performing anticoagulant therapy, $29 \%$ antiplatelet agents and $4 \%$ both therapies. Of those with low thrombotic risk, $63.6 \%$ was wrongly performing some kind of antithrombotic therapy; among patients with high risk or valvular disease $56.8 \%$ was properly undergoing anticoagulant therapy.

Conclusion: The prevalence of atrial fibrillation as well as the frequency of the main comorbidities associated with it are in line with the majority of studies. Although most patients are undergoing oral anticoagulation, only $56.8 \%$ of those with atrial fibrillation was performing adequate antithrombotic therapy as recommended by the European Society of Cardiology guidelines, which denote a marked underutilization of this treatment.

Keywords: Atrial Fibrillation; Fibrinolytic Agents; Stroke; Primary Health Care; Portugal.

\section{INTRODUÇÃO}

A fibrilhação auricular (FA) é a arritmia sustentada mais prevalente na prática clínica, com prevalências globais nos países desenvolvidos na ordem de 1,5-2\%, ${ }^{1}$ admitindo-se, contudo, que esta possa não ser similar em todos os países. ${ }^{2}$ Existem dois estudos publicados que determinaram a prevalência de FA na população portuguesa: em 2003, a Rede dos Médicos Sentinela determinou uma prevalência de FA de $0,53 \%$ nos Cuidados de Saúde Primários (CSP), ${ }^{3}$ o estudo FAMA, publicado em 2010, estimou que a prevalência de FA na população portuguesa com idade $\geq 40$ anos era de $2,5 \%{ }^{4} \mathrm{~A}$ prevalência aumenta com a idade estimando-se que aumente pelo menos para o dobro nos próximos 50 anos acompanhando o envelhecimento da população. ${ }^{5}$ Sendo uma doença directamente associada à idade, estima-se que a sua incidência duplique por cada década de vida a partir dos $50 a^{2} \operatorname{s}^{2}$ e que uma em cada quatro pessoas aos 40 anos desenvolva FA. ${ }^{6}$

Para além da idade, associam-se a um maior risco de FA o género masculino, a história familiar de FA, o alcoolismo, a obesidade, o hipertiroidismo, o tromboembolismo

1. Unidade de Saúde Familiar Nova Via. ACeS Grande Porto VIII Espinho/Gaia. Vila Nova de Gaia. Portugal.

Recebido: 03 de Junho de 2014 - Aceite: 21 de Janeiro de 2015 | Copyright @ Ordem dos Médicos 2015 
pulmonar (TEP) e diversas patologias cardiovasculares. Destas, destacam-se a insuficiência cardíaca congestiva (ICC); a hipertensão arterial (HTA), especialmente quando associada a hipertrofia do ventrículo esquerdo (HVE); a miocardiopatia hipertrófica ou dilatada; a doença coronária (DC), especialmente se antecedentes de enfarte agudo do miocárdio (EAM); a doença valvular, mais frequentemente da válvula mitral, e a diabetes mellitus (DM). ${ }^{2}$

Nos últimos 20 anos as admissões hospitalares por FA têm aumentado cerca de $66 \%$ devido ao envelhecimento da população, ao aumento da prevalência de doenças cardíacas crónicas e ao maior uso da monitorização electrocardiográfica ambulatória, ${ }^{7}$ traduzindo-se numa causa cada vez mais importante de despesa com a saúde. ${ }^{8} \mathrm{~A}$ maior parte dos custos deve-se a internamentos hospitalares. $\mathrm{Na}$ presença de FA, os internamentos são duas vezes mais frequentes, os internamentos múltiplos são três vezes mais frequentes e os internamentos por motivo cardiovascular são quatro vezes mais frequentes. ${ }^{9}$ Por outro lado, a medicação dos doentes com FA representa uma pequena parcela dos custos da doença. ${ }^{10}$

A FA é classificada em 1) primeiro episódio diagnosticado de FA - quando a arritmia é detectada pela primeira vez, independentemente da sua duração; 2) FA recorrente paroxística - se a arritmia termina espontaneamente e tem duração inferior a sete dias (geralmente inferior a 48 horas); 3) FA recorrente persistente - se persiste para além de sete dias, podendo ser de longa duração (se persistir por mais de um ano); 4) FA persistente - quando a arritmia se torna permanente, com diagnóstico efectuado há anos e com cardioversão sem sucesso ou não tentada..$^{5} \mathrm{O}$ termo FA isolada aplica-se aos indivíduos com menos de 60 anos sem evidência clínica ou ecocardiográfica de doença cardiopulmonar, incluindo HTA. ${ }^{5} \mathrm{O}$ termo FA não valvular aplica-se aos casos de FA sem doença mitral reumática e sem prótese ou reparação valvular. ${ }^{5}$

A FA pode provocar alterações hemodinâmicas importantes mas o seu prognóstico é marcado, sobretudo, pelos fenómenos tromboembólicos a que está associada, com consequências significativas em termos de morbilidade e mortalidade. Também constitui um factor de risco independente quer para a mortalidade global, quer para a morte súbita de causa cardíaca. ${ }^{2}$

As embolias relacionadas com a FA são responsáveis por $15 \%$ dos acidentes vasculares cerebrais (AVC) ${ }^{11}$, que têm maior gravidade e pior prognóstico. ${ }^{12} \mathrm{~A}$ incidência de AVC na FA não reumática é de cerca de $5 \%$ ao ano (quatro a cinco vezes maior que a da população em geral para qualquer grupo etário), ${ }^{11,13}$ aumentando para 13 a $14 \%$ na FA com valvulopatia concomitante. ${ }^{14} \mathrm{~A}$ proporção de AVC isquémicos atribuíveis à $\mathrm{FA}$ também aumenta com a idade: $1,5 \%$ dos 50 aos 59 anos de idade e $23,5 \%$ dos 80 aos 89 anos. $^{14}$

Uma vez que a prevalência da HTA ou da hipercolesterolemia em Portugal tem valores semelhantes, senão mesmo inferiores, relativamente aos restantes países da União Europeia, a elevada taxa de AVC em Portugal pode ficar a dever-se à existência de uma prevalência elevada de FA, frequentemente tratada de modo inadequado. ${ }^{2}$

Está provada a eficácia da anticoagulação oral com antagonistas da vitamina $\mathrm{K}$, como a varfarina, na prevenção do AVC em doentes com FA (diminuição do risco de AVC em $64 \%$ em comparação com o placebo)..$^{15}$ Quanto à antiagregação plaquetária com ácido acetilsalicílico (AAS), a sua eficácia é mais modesta (diminuição do risco de AVC não fatal em $21 \%$ versus placebo). ${ }^{16}$ Outros antiagregantes plaquetários não são recomendados. ${ }^{7}$ Contudo, a antiagregação dupla com AAS e clopidogrel tem uma eficácia superior à monoterapia com AAS, embora o risco de hemorragia também seja superior ao da monoterapia com AAS bem como da anticoagulação oral, ${ }^{17}$ devendo esta opção ficar reservada apenas para os doentes que recusem qualquer tipo de anticoagulação e a monoterapia com AAS reservada para aqueles que recusem qualquer tipo de anticoagulação e não possam tolerar a antiagregação dupla com AAS e clopidogrel por elevado risco de hemorragia. Não existe benefício na combinação de anticoagulantes orais (ACO) com antiagregantes plaquetários relativamente à terapêutica individual com ACO em dose ajustada. ${ }^{7}$

Mais recentemente têm surgido novas estratégias terapêuticas para a prevenção tromboembólica na FA, como os inibidores directos da trombina, como o dabigatrano, e os inibidores do factor $\mathrm{Xa}$, como o rivaroxabano e o apixabano. Estes novos fármacos têm mostrado ser superiores na prevenção trombótica comparativamente à varfarina, ${ }^{18,19}$ apresentam uma redução marcada da incidência de hemorragia intracraniana ${ }^{20}$ e têm a vantagem de serem tomados diariamente numa dose fixa e não necessitarem de controlo periódico dos níveis da Razão Normalizada Internacional (International Normalized Ratio - INR). Apresentam também algumas desvantagens: necessitam de ajuste de dose mediante a função renal, exigem uma boa adesão terapêutica e ainda não possuem um antídoto específico no caso de hemorragia. ${ }^{1}$

Contudo, apesar da forte evidência do benefício do uso de ACO na prevenção do AVC na maioria dos doentes com FA, infelizmente esta terapêutica é largamente subutilizada e muitas vezes os níveis da INR ficam abaixo dos valores terapêuticos no caso da varfarina. ${ }^{21}$

A estratificação do risco tromboembólico é um componente crítico da avaliação clínica do doente com FA, constituindo um indicador da qualidade dos cuidados de saúde prestados a estes doentes. Na recente actualização das suas recomendações para a orientação da FA, a European Society of Cardiology (ESC) passou a recomendar vivamente que haja uma mudança na prática clínica no sentido de se identificar os doentes com FA com 'verdadeiro baixo risco' (i.e. 'idade < 65 anos e presença de FA isolada', que não necessitam de qualquer terapia antitrombótica), em vez de se tentar identificar os doentes de 'alto risco'.' Assim, em todas as situações de FA valvular, como o risco anual de complicações tromboembólicas é muito elevado, a terapêutica com ACO é obrigatória, ${ }^{1}$ nos doentes com FA não valvular, o risco anual de complicações tromboembó- 
licas é muito variável, sendo necessário calcular o grau de risco tromboembólico com o objectivo de se identificarem os doentes com FA com 'verdadeiro baixo risco', que não irão necessitar de terapêutica antitrombótica. A ESC recomenda o uso da ferramenta $\mathrm{CHA}_{2} \mathrm{DS}_{2}$-VASc para o cálculo deste risco. ${ }^{1,5}$

Além do risco tromboembólico, o risco hemorrágico também deve ser calculado e avaliado. A ESC recomenda o uso da ferramenta HAS-BLED. ${ }^{1,5}$ No entanto, o resultado desta ferramenta só por si não deve ser usado para excluir a terapêutica anticoagulante. A utilidade deste resultado passa por permitir uma correcta avaliação do risco hemorrágico do doente com $\mathrm{FA}$, identificando os factores de risco para hemorragia em cada caso, permitindo, assim, intervir no seu controlo.

Sendo o AVC a principal causa de mortalidade e incapacidade por doenças cardiovasculares em Portugal ${ }^{7}$ e a FA um dos seus importantes factores de risco, justifica-se a importância de todas as medidas tendentes à sua prevenção. Tendo em conta que as doenças do aparelho circulatório são a primeira causa de morte nos Agrupamentos de Centros de Saúde (ACeS) de Gaia e Espinho/Gaia (no triénio 2008-2010), ${ }^{22}$ torna-se fundamental conhecer a prevalência local desta patologia e estudar a população afectada de modo a melhorar estratégias de saúde.

O objectivo primário deste estudo foi determinar a prevalência de FA conhecida numa população de utentes inscritos em oito Unidades de Saúde Familiar (USF) do conceIho de Vila Nova de Gaia.

Os objectivos secundários foram: a) quantificar as principais comorbilidades associadas à FA; b) identificar a terapêutica antitrombótica em curso; c) avaliar a adequação da terapêutica antitrombótica segundo as recomendações de 2012 da ESC. ${ }^{1}$

\section{MATERIAL E MÉTODOS}

Estudo observacional, transversal e analítico, que decorreu em oito USF do concelho de Vila Nova de Gaia: USF Arco do Prado, USF Nova Salus, USF Saúde no Futuro (unidades de saúde pertencentes ao ACeS Grande Porto VII - Gaia), USF Além D'Ouro, USF Canelas, USF Nova Via, USF São Félix da Marinha e USF São Miguel (unidades de saúde pertencentes ao ACeS Grande Porto VIII Espinho/Gaia), tendo sido obtida autorização de ambos os ACeS bem como parecer favorável da Comissão de Ética da Administração Regional de Saúde (ARS) do Norte.

A população estudada corresponde a todos os utentes com idade igual ou superior a 30 anos inscritos nas oito USF. Foram considerados casos de FA todos os utentes com o diagnóstico de FA registado no respectivo processo clínico, aferido através da presença do código K78 - Fibrilhação/Flutter auricular da International Classification of Primary Care $2^{\text {nd }}$ edition (ICPC-2), ${ }^{23}$ na lista de problemas do utente.

As variáveis estudadas foram:

- Idade

- Género
- Comorbilidades associadas à FA - classificada em "Presente" ou "Ausente", consoante a presença ou ausência das seguintes comorbilidades: HTA, ICC, AVC ou acidente isquémico transitório (AIT) prévio, DM, estenose mitral ou trombo intracavitário, fracção de ejecção ventricular esquerda (FEVE) $\leq 40 \%$, EAM, doença arterial periférica (DAP) e placas ateromatosas na aorta. Estes diagnósticos/problemas foram identificados recorrendo à lista de problemas do processo clínico electrónico e/ou registo do resultado de ecocardiograma.

- Risco trombótico - resultado calculado através da ferramenta $\mathrm{CHA}_{2} \mathrm{DS}_{2}$-VASc.

- Terapêutica antiagregante - classificada em 'AAS', 'clopidogrel', 'ticlopidina', 'triflusal', 'dipiridamol', 'ticagrelor', 'nenhum' ou 'desconhecido', consoante a terapêutica antiagregante que o utente fazia à data da última consulta na sua USF.

- Terapêutica anticoagulante - classificada em 'acenocumarol', 'varfarina', 'dabigatrano', 'rivaroxabano', 'nenhum' ou 'desconhecido', consoante a terapêutica anticoagulante que o utente fazia à data da última consulta na sua USF.

Para a obtenção da lista de todos os utentes com o diagnóstico de FA foi utilizado o Módulo de Informação e Monitorização das Unidades Funcionais (MIM@UF ${ }^{\circledR}$ ). Os restantes dados foram colhidos do processo clínico electrónico cujas fontes foram o programa informático Sistema de Apoio ao Médico (SAM) e a Plataforma de Dados da Saúde (PDS).

Os dados colhidos foram codificados e registados numa base de dados informática criada pelos autores para o efeito no programa Microsoft Excel ${ }^{\circledR} 2010$.

Foi dispensado o consentimento informado, uma vez que não foram colhidos nem registados quaisquer elementos identificativos dos utentes. Além disso, sendo este um estudo de prevalência, e não de intervenção, não interferiu directa ou indirectamente com o doente.

O tratamento estatístico dos dados foi efectuado com recurso aos programas informáticos Microsoft Excel ${ }^{\circledR} 2010$ e SPSS ${ }^{\circledR}$ versão 20.0. A análise descritiva dos dados teve por base a distribuição de frequências e as medidas de tendência central e de dispersão absoluta. A análise inferencial dos dados foi feita recorrendo a tabelas de contingência e ao teste de Qui-quadrado, tendo sido usado um nível de significância de 5\%.

\section{RESULTADOS}

Estudamos 73423 utentes com idades compreendidas entre os 30 e os 103 anos, dos quais $46,4 \%$ do género masculino. Destes, 944 apresentavam o diagnóstico de FA (código K78 - Fibrilhação/Flutter auricular, da ICPC-2), com idades compreendidas entre os 33 e os 100 anos (média de 74,7 anos, desvio padrão de 9,95 e mediana de 76 anos), dos quais $50,4 \%$ eram do género masculino.

A prevalência calculada de FA conhecida na população foi de $1,29 \%$, sendo superior no género masculino - 1,40\% 
versus $1,19 \%$ - (Tabela 1$)$, diferença esta estatisticamente significativa $(p=0,01)$.

Também se verificou um aumento da prevalência de FA com a idade $(p<0,001)$ (Tabela 1$)$.

A HTA foi a comorbilidade mais frequente com $76,4 \%$, seguida da ICC $(32,0 \%)$ e da DM $(28,2 \%) ; 15,9 \%$ dos doentes presentavam antecedentes de AVC ou AIT e 9,5\% teve um EAM. A DAP/placa ateromatosa da aorta estava presente em $8,1 \%$ dos utentes e a doença valvular em $7,3 \%$ dos utentes; $3,1 \%$ dos utentes tinha uma fracção de ejecção ventricular esquerda igual ou inferior a $40 \%$. Apenas um doente apresentava registo de trombo intracavitário.

Excluindo os 69 utentes que apresentavam doença valvular, que representa, por si só, elevado risco trombótico, calculámos o risco trombótico através da ferramenta $\mathrm{CHA}_{2} \mathrm{DS}_{2}$-VASc. Verificámos que 2,9\% dos utentes apresentava baixo risco (score $=0$ ), enquanto $7,4 \%$ apresentava score $=1$ e $89,6 \%$ score $\geq 2$.

Relativamente à distribuição de acordo com a terapêutica antitrombótica verificámos que $52 \%$ dos doentes fazia terapêutica anticoagulante, $29 \%$ fazia antiagregantes plaquetários e $4 \%$ fazia terapêutica com anticoagulantes orais em conjunto com antiagregantes; $7 \%$ dos doentes não fazia qualquer terapêutica antitrombótica e em $8 \%$ dos doentes a terapêutica era desconhecida.
Especificando os ACO usados, verificámos que a varfarina era o anticoagulante mais prescrito (75,4\%); $20,6 \%$ dos utentes estava medicado com acenocumarol e 4,1\% com dabigatrano; nenhum utente estava medicado com rivaroxabano. Relativamente aos antiagregantes plaquetários, $76,2 \%$ dos utentes estava medicado com AAS, $13,6 \%$ com clopidogrel, $7,2 \%$ com triflusal, $1,5 \%$ com dipiridamol e $1,5 \%$ com ticlopidina; nenhum utente estava medicado com ticagrelor.

Uma vez que a indicação para terapêutica antitrombótica depende do risco trombótico individual, calculado através da ferramenta $\mathrm{CHA}_{2} \mathrm{DS}_{2}-\mathrm{VASc}$, investigámos a adequação da medicação antitrombótica instituída segundo as mais recentes recomendações da ESC emitidas em 2012.1

Dos 25 utentes com score $=0$ (utentes com 'verdadeiro baixo risco' e sem indicação para qualquer terapêutica antitrombótica) a terapêutica era desconhecida em 3. A Tabela 2 apresenta a terapêutica antitrombótica dos utentes com FA com score $=0$.

Dos 919 utentes com elevado risco trombótico $(97,4 \%)$ e, portanto, com indicação para terapêutica antitrombótica (utentes com FA com doença valvular e utentes com FA não valvular com score $\mathrm{CHA}_{2} \mathrm{DS}_{2}-\mathrm{VASc} \geq 1$ ), verificámos que apenas $56,8 \%$ destes utentes estava adequadamente hipocoagulado (Tabela 3).

Tabela 1 - Prevalência de FA conhecida por género e faixa etária

\begin{tabular}{|c|c|c|c|c|c|c|c|c|c|c|c|}
\hline & & & Homens & & & Mulhere & & & Total & & \\
\hline & & População & $\begin{array}{c}\mathrm{N} . .^{\circ} \\
\text { Casos }\end{array}$ & $\begin{array}{c}\text { Taxa \% } \\
\text { (IC 95\%) }\end{array}$ & População & $\begin{array}{c}\mathrm{N}^{\circ} \\
\text { Casos }\end{array}$ & $\begin{array}{c}\text { Taxa \% } \\
\text { (IC 95\%) }\end{array}$ & População & $\begin{array}{c}\text { N. }{ }^{\circ} \\
\text { Casos }\end{array}$ & $\begin{array}{c}\text { Taxa \% } \\
\text { (IC 95\%) }\end{array}$ & $p$ \\
\hline & $30-39$ & 8173 & 2 & $\begin{array}{c}0,02 \\
(0-0,05)\end{array}$ & 8904 & 0 & $\begin{array}{c}0,00 \\
-\end{array}$ & 17077 & 2 & $\begin{array}{c}0,01 \\
(0-0,02)\end{array}$ & \\
\hline & $40-49$ & 8397 & 14 & $\begin{array}{c}0,17 \\
(0,08-0,26)\end{array}$ & 9208 & 3 & $\begin{array}{c}0,03 \\
(0-0,07)\end{array}$ & 17605 & 17 & $\begin{array}{c}0,10 \\
(0,05-0,15)\end{array}$ & \\
\hline Faixa & $50-59$ & 7096 & 36 & $\begin{array}{c}0,51 \\
(0,34-0,68)\end{array}$ & 7879 & 19 & $\begin{array}{c}0,24 \\
(0,13-0,35)\end{array}$ & 14975 & 55 & $\begin{array}{c}0,37 \\
(0,27-0,47)\end{array}$ & \\
\hline $\begin{array}{l}\text { etária } \\
\text { (anos) }\end{array}$ & $60-69$ & 5532 & 98 & $\begin{array}{c}1,77 \\
(1,42-2,12)\end{array}$ & 6239 & 72 & $\begin{array}{c}1,15 \\
(0,89-1,41)\end{array}$ & 11771 & 170 & $\begin{array}{c}1,44 \\
(1,22-1,66)\end{array}$ & $<0,001$ \\
\hline & $70-79$ & 3328 & 188 & $\begin{array}{c}5,65 \\
(4,87-6,43)\end{array}$ & 4244 & 181 & $\begin{array}{c}4,26 \\
(3,65-4,87)\end{array}$ & 7572 & 369 & $\begin{array}{c}4,87 \\
(4,39-5,35)\end{array}$ & \\
\hline & $\geq 80$ & 1557 & 138 & $\begin{array}{c}8,86 \\
(7,45-10,27)\end{array}$ & 2866 & 193 & $\begin{array}{c}6,73 \\
(5,81-7,65)\end{array}$ & 4423 & 331 & $\begin{array}{c}7,48 \\
(6,70-8,26)\end{array}$ & \\
\hline Tc & & 34083 & 476 & $\begin{array}{c}1,40 \\
(1,28-1,52)\end{array}$ & 39340 & 468 & $\begin{array}{c}1,19 \\
(1,08-1,30)\end{array}$ & 73423 & 944 & $\begin{array}{c}1,29 \\
(1,21-1,37)\end{array}$ & \\
\hline & & & & 0 & & & & & & & \\
\hline
\end{tabular}

FA - Fibrilhação auricular; IC - Intervalo de confiança.

Tabela 2 - Distribuição dos utentes com FA com score $\mathrm{CHA}_{2} \mathrm{DS}_{2}-\mathrm{VASc}=0$ segundo a terapêutica antitrombótica realizada

\begin{tabular}{|c|c|c|c|}
\hline & Com antiagregante plaquetário & Sem antiagregante plaquetário & TOTAL \\
\hline Com ACO & $\begin{array}{c}1 \\
(4,5 \%)\end{array}$ & $\begin{array}{c}5 \\
(22,7 \%)\end{array}$ & $\begin{array}{c}6 \\
(27,2 \%)\end{array}$ \\
\hline Sem ACO & $\begin{array}{c}8 \\
(36,4 \%)\end{array}$ & $\begin{array}{c}8 \\
(36,4 \%)\end{array}$ & $\begin{array}{c}16 \\
(72,8 \%)\end{array}$ \\
\hline TOTAL & $\begin{array}{c}9 \\
(40,9 \%)\end{array}$ & $\begin{array}{c}13 \\
(59,1 \%)\end{array}$ & $\begin{array}{c}22 \\
(100 \%)\end{array}$ \\
\hline
\end{tabular}

FA - Fibrilhação auricular; ACO - Anticoagulante oral. 
Tabela 3 - Distribuição dos doentes com FA com elevado risco trombótico, de acordo com o tipo de terapêutica antitrombótica realizada

\begin{tabular}{|c|c|c|c|}
\hline & $\begin{array}{c}\text { Score } \\
\mathrm{CHA}_{2} \mathrm{DS}_{2}-\mathrm{VASc} \geq 1\end{array}$ & FA com doença valvular & Total \\
\hline Ausência de tratamento & $\begin{array}{c}50 \\
(5,9 \%)\end{array}$ & $\begin{array}{c}5 \\
(5,8 \%)\end{array}$ & $\begin{array}{c}55 \\
(6,0 \%)\end{array}$ \\
\hline ACO & $\begin{array}{c}422 \\
(49,7 \%)\end{array}$ & $\begin{array}{c}44 \\
(63,8 \%)\end{array}$ & $\begin{array}{c}466 \\
(50,7 \%)\end{array}$ \\
\hline Antiagregante plaquetário & $\begin{array}{c}242 \\
(28,5 \%)\end{array}$ & $\begin{array}{c}13 \\
(18,8 \%)\end{array}$ & $\begin{array}{c}255 \\
(27,7 \%)\end{array}$ \\
\hline Antiagregação plaquetária dupla & $\begin{array}{c}9 \\
(1,1 \%)\end{array}$ & $\begin{array}{c}4 \\
(5,8 \%)\end{array}$ & $\begin{array}{c}13 \\
(1,4 \%)\end{array}$ \\
\hline ACO + Antiagregante plaquetário & $\begin{array}{c}54 \\
(6,3 \%)\end{array}$ & $\begin{array}{c}2 \\
(2,9 \%)\end{array}$ & $\begin{array}{c}56 \\
(6,1 \%)\end{array}$ \\
\hline Desconhecido & $\begin{array}{c}72 \\
(8,5 \%)\end{array}$ & $\begin{array}{c}2 \\
(2,9 \%)\end{array}$ & $\begin{array}{c}74 \\
(8,1 \%)\end{array}$ \\
\hline Total & $\begin{array}{c}849 \\
(100 \%)\end{array}$ & $\begin{array}{c}70 \\
(100 \%)\end{array}$ & $\begin{array}{c}919 \\
(100 \%)\end{array}$ \\
\hline
\end{tabular}

FA - Fibrilhação auricular; ACO - Anticoagulante oral.

Tabela 4 - Distribuição dos doentes com FA por Score $\mathrm{CHA}_{2} \mathrm{DS}_{2}$-VASc, de acordo com o tipo de terapêutica antitrombótica utilizada

Tipo de terapêutica antitrombótica

\begin{tabular}{|c|c|c|c|c|c|}
\hline $\mathrm{CHA}_{2} \mathrm{DS}_{2}-\mathrm{VASc}$ & $\begin{array}{c}\text { Sem } \\
\text { medicação }\end{array}$ & $\begin{array}{c}\text { Com } \\
\text { medicação }\end{array}$ & ACO & $\begin{array}{l}\text { Antiagregante } \\
\text { plaquetário }\end{array}$ & $\begin{array}{c}\text { ACO + Antiagregante } \\
\text { plaquetário e/ou dupla } \\
\text { antiagregação }\end{array}$ \\
\hline 1 & $\begin{array}{c}8 \\
(13,1 \%)\end{array}$ & $\begin{array}{c}53 \\
(86,9 \%)\end{array}$ & $\begin{array}{c}27 \\
(44,3 \%)\end{array}$ & $\begin{array}{c}20 \\
(32,8 \%)\end{array}$ & $\begin{array}{c}6 \\
(9,8 \%)\end{array}$ \\
\hline 2 & $\begin{array}{c}15 \\
(14,0 \%)\end{array}$ & $\begin{array}{c}92 \\
(86,0 \%)\end{array}$ & $\begin{array}{c}52 \\
(48,6 \%)\end{array}$ & $\begin{array}{c}32 \\
(29,9 \%)\end{array}$ & $\begin{array}{c}8 \\
(7,5 \%)\end{array}$ \\
\hline 3 & $\begin{array}{c}15 \\
(9,4 \%)\end{array}$ & $\begin{array}{c}145 \\
(90,6 \%)\end{array}$ & $\begin{array}{c}97 \\
(60,6 \%)\end{array}$ & $\begin{array}{c}35 \\
(21,9 \%)\end{array}$ & $\begin{array}{c}13 \\
(8,1 \%)\end{array}$ \\
\hline 4 & $\begin{array}{c}6 \\
(2,9 \%)\end{array}$ & $\begin{array}{c}204 \\
(97,1 \%)\end{array}$ & $\begin{array}{c}112 \\
(53,3 \%)\end{array}$ & $\begin{array}{c}79 \\
(37,6 \%)\end{array}$ & $\begin{array}{c}13 \\
(6,2 \%)\end{array}$ \\
\hline 5 & $\begin{array}{c}5 \\
(4,0 \%)\end{array}$ & $\begin{array}{c}119 \\
(96,0 \%)\end{array}$ & $\begin{array}{c}67 \\
(54,0 \%)\end{array}$ & $\begin{array}{c}38 \\
(30,6 \%)\end{array}$ & $\begin{array}{c}14 \\
(11,3 \%)\end{array}$ \\
\hline 6 & $\begin{array}{c}1 \\
(1,5 \%)\end{array}$ & $\begin{array}{c}65 \\
(98,5 \%)\end{array}$ & $\begin{array}{c}39 \\
(59,1 \%)\end{array}$ & $\begin{array}{c}21 \\
(31,8 \%)\end{array}$ & $\begin{array}{c}5 \\
(7,6 \%)\end{array}$ \\
\hline 7 & $\begin{array}{c}0 \\
(0 \%)\end{array}$ & $\begin{array}{c}33 \\
(100 \%)\end{array}$ & $\begin{array}{c}17 \\
(51,5 \%)\end{array}$ & $\begin{array}{c}12 \\
(36,4 \%)\end{array}$ & $\begin{array}{c}4 \\
(12,1 \%)\end{array}$ \\
\hline 8 & $\begin{array}{c}0 \\
(0 \%)\end{array}$ & $\begin{array}{c}13 \\
(100 \%)\end{array}$ & $\begin{array}{c}9 \\
(69,2 \%)\end{array}$ & $\begin{array}{c}4 \\
(30,8 \%)\end{array}$ & $\begin{array}{c}0 \\
(0 \%)\end{array}$ \\
\hline 9 & $\begin{array}{c}0 \\
(0 \%)\end{array}$ & $\begin{array}{c}3 \\
(100 \%)\end{array}$ & $\begin{array}{c}2 \\
(66,7 \%)\end{array}$ & $\begin{array}{c}1 \\
(33,3 \%)\end{array}$ & $\begin{array}{c}0 \\
(0 \%)\end{array}$ \\
\hline
\end{tabular}

FA - Fibrilhação auricular; ACO - Anticoagulante oral.

Este valor sobe para $57,7 \%$ se excluirmos os utentes com score $=1$, nos quais a terapêutica antitrombótica é recomendada mas cuja indicação não é absoluta. Contudo, dada a esta terapêutica ser recomendada igualmente nestes doentes, ao longo deste trabalho os utentes com score $=1$ foram sempre considerados doentes de elevado risco trombótico.

Correlacionando o score $\mathrm{CHA}_{2} \mathrm{DS}_{2}$-VASc com a terapêutica antitrombótica utilizada, é possível verificar que quanto maior o score maior a probabilidade de o doente se encontrar medicado (Tabela 4). Contudo, não é possível estabelecer uma relação entre o score e o tipo de terapêu- tica antitrombótica utilizada (Tabela 4).

Verificámos que a idade também influencia a decisão de instituir terapêutica antitrombótica, ou seja, com o avançar da idade aumenta a utilização de terapêutica antitrombótica (Tabela 5). Contudo, neste caso também não é possível estabelecer uma relação entre a idade e o tipo de terapêutica antitrombótica utilizada, apesar de se verificar uma clara preferência pelos antiagregantes plaquetários nos extremos etários (nos mais jovens com uma taxa de $71,4 \%$ e nos mais idosos, com menor expressão, totalizando $35,4 \%$ ) (Tabela 5). 
Tabela 5 - Distribuição dos doentes com FA com elevado risco trombótico por faixa etária, de acordo com o tipo de terapêutica antitrombótica utilizada

Tipo de terapêutica antitrombótica

\begin{tabular}{|c|c|c|c|c|c|}
\hline $\begin{array}{c}\text { Faixa etária } \\
\text { (anos) }\end{array}$ & Sem medicação & $\begin{array}{c}\text { Com } \\
\text { medicação }\end{array}$ & $\mathrm{ACO}$ & $\begin{array}{l}\text { Antiagregante } \\
\text { plaquetário }\end{array}$ & $\begin{array}{l}\text { ACO + Antiagregante } \\
\text { plaquetário elou dupla } \\
\text { antiagregação }\end{array}$ \\
\hline $40-49$ & $\begin{array}{c}2 \\
(28,6 \%)\end{array}$ & $\begin{array}{c}5 \\
(71,4)\end{array}$ & $\begin{array}{c}0 \\
(0 \%)\end{array}$ & $\begin{array}{c}5 \\
(71,4 \%)\end{array}$ & $\begin{array}{c}0 \\
(0 \%)\end{array}$ \\
\hline $50-59$ & $\begin{array}{c}6 \\
(13,0 \%)\end{array}$ & $\begin{array}{c}40 \\
(87,0 \%)\end{array}$ & $\begin{array}{c}27 \\
(58,7 \%)\end{array}$ & $\begin{array}{c}9 \\
(19,6 \%)\end{array}$ & $\begin{array}{c}4 \\
(8,7 \%)\end{array}$ \\
\hline $60-69$ & $\begin{array}{c}16 \\
(10,4 \%)\end{array}$ & $\begin{array}{c}138 \\
(89,6 \%)\end{array}$ & $\begin{array}{c}88 \\
(57,1 \%)\end{array}$ & $\begin{array}{c}41 \\
(26,6 \%)\end{array}$ & $\begin{array}{c}9 \\
(5,8 \%)\end{array}$ \\
\hline $70-79$ & $\begin{array}{c}16 \\
(4,8 \%)\end{array}$ & $\begin{array}{c}320 \\
(95,2 \%)\end{array}$ & $\begin{array}{c}198 \\
(58,9 \%)\end{array}$ & $\begin{array}{c}94 \\
(28,0 \%)\end{array}$ & $\begin{array}{c}28 \\
(8,3 \%)\end{array}$ \\
\hline$\geq 80$ & $\begin{array}{c}15 \\
(4,9 \%)\end{array}$ & $\begin{array}{c}287 \\
(95,0 \%)\end{array}$ & $\begin{array}{c}153 \\
(50,7 \%)\end{array}$ & $\begin{array}{c}106 \\
(35,1 \%)\end{array}$ & $\begin{array}{c}28 \\
(9,3 \%)\end{array}$ \\
\hline
\end{tabular}

FA - Fibrilhação auricular; ACO - Anticoagulante oral.

\section{DISCUSSÃO}

A prevalência global de FA encontrada neste estudo foi superior à encontrada no estudo da Rede Médicos-Sentinela ${ }^{3}$ mas inferior ao estudo FAMA, ${ }^{4}$ que apresentaram prevalências globais de $0,53 \%$ e $2,5 \%$ respectivamente. Contudo, importa referir que o estudo da Rede Médicos-Sentinela ${ }^{3}$ apresenta uma metodologia de recolha de dados semelhante à usada neste estudo - onde a prevalência é estimada com base nos casos de FA já diagnosticados, ou seja, FA conhecida - enquanto no estudo FAMA ${ }^{4}$ foi usada uma amostra representativa da população portuguesa onde foi realizado o estudo para diagnosticar FA e assim estimar a sua prevalência. É também de ressalvar que no estudo da Rede Médicos-Sentinela ${ }^{3}$ a população estudada incluiu todos os indivíduos de todas as idades (incluindo crianças), enquanto a população estudada no estudo FAMA ${ }^{4}$ incluiu apenas indivíduos com idade igual ou superior a 40 anos e no presente estudo foram usados todos os indivíduos com idades a partir dos 30 anos (idade a partir da qual se identificou o caso mais jovem de FA). Se considerarmos apenas a população com idade igual ou superior a 35 anos no estudo dos Médicos Sentinela, calcula-se uma prevalência de FA de 0,94\%.

Relativamente a estudos internacionais, as prevalências de FA também variam, nomeadamente de acordo com a metodologia utilizada: na população abrangida pelo sistema de seguros estatal dos Estados Unidos da América em $2009,{ }^{24}$ que compreendia os indivíduos com idade superior a 20 anos, foi encontrada uma prevalência de 1,12\%; no estudo ATRIA ${ }^{25}$ a prevalência rondou os $0,95 \%$ numa população adulta a partir dos 20 anos de idade; no estudo espanhol OFRECE, ${ }^{26}$ que segue uma metodologia semelhante ao estudo FAMA, ${ }^{4}$ a prevalência da FA rondou os $4,4 \%$ e superou as estimativas até agora existentes e que indicavam a possibilidade de a prevalência de FA poder ocorrer até aos $2 \%$ nos países desenvolvidos. ${ }^{1}$

Comparando a nossa prevalência com as prevalências de FA do estudo FAMA ${ }^{4}$ e OFRECE ${ }^{26}$ (que procuraram activamente os casos de FA na população estudada com recurso ao electrocardiograma) - 1,29\% versus $2,5 \%, 4,4 \%$ respectivamente - poderá existir um possível subdiagnóstico da FA na nossa população.

Reconhece-se a FA como uma patologia que surge com o aumentar da idade,${ }^{1}$ e este estudo mostrou que existe um aumento da prevalência de FA com a idade e que este foi estatisticamente significativo, de acordo com o observado nos estudos da rede Médicos-Sentinela, ${ }^{3}$ estudo FAMA, ${ }^{4}$ estudo de Naccarelli, ${ }^{24}$ estudo ATRIA ${ }^{25}$ e no estudo OFRECE. ${ }^{26}$

Também se observou uma superioridade estatisticamente significativa da prevalência de FA nos indivíduos do género masculino $(1,40 \%$ versus $1,29 \%, p=0,01)$, estando de acordo com o estudo da Rede Médicos-Sentinela ${ }^{3}$ e com o estudo ATRIA. ${ }^{25}$ Neste, para além do aumento da prevalência da FA no género masculino $(1,1 \%$ versus $0,8 \%, p<$ $0,01)$ mostrou ainda que esta diferença é observada em todas as faixas etárias estudadas, tal como demonstramos no presente estudo. Os estudos $\mathrm{FAMA}^{4}$ e $\mathrm{OFRECE}^{26}$ não encontraram diferenças na prevalência de FA entre os géneros.

Relativamente às comorbilidades estudadas, também encontrámos diferenças relativamente aos estudos consultados (Tabela 6): no nosso estudo a HTA estava presente em $76,4 \%$ dos indivíduos com $\mathrm{FA}$, frequência superior à maioria dos estudos consultados; a frequência de ICC encontrada no nosso estudo foi inferior ao estudo da Rede Médicos-Sentinela ${ }^{3}$ e semelhante à encontrada em estudos internacionais nomeadamente o estudo ATRIA, ${ }^{25} \mathrm{o}$ estudo de Naccarelli et $\mathrm{al}^{24} \mathrm{e}$ o estudo OFRECE, ${ }^{26}$ em relação à $\mathrm{DM}$, a sua frequência foi superior à do estudo da Rede Médicos-Sentinela, ${ }^{3}$ do estudo FAMA ${ }^{4}$ e do estudo ATRIA ${ }^{25}$ e foi semelhante ao observado nos estudos de Naccarelli ${ }^{24} \mathrm{e}$ OFRECE. ${ }^{26}$

Utilizámos a ferramenta $\mathrm{CHA}_{2} \mathrm{DS}_{2}$-Vasc na avaliação 
Tabela 6 - Comparação das comorbilidades presentes nos doentes com FA diversos estudos analisados

\begin{tabular}{|c|c|c|c|c|c|}
\hline Estudo Comorbilidades & HTA & DM & ICC & $\begin{array}{l}\text { Doença } \\
\text { Valvular }\end{array}$ & $\begin{array}{l}\text { AVC/AIT } \\
\text { anterior }\end{array}$ \\
\hline FATA & $76,4 \%$ & $28,2 \%$ & $32,0 \%$ & $7,3 \%$ & $15,9 \%$ \\
\hline FAMA $^{4}$ & $71 \%$ & $26,4 \%$ & NA & NA & NA \\
\hline Rede Médicos Sentinela ${ }^{3}$ & $70,8 \%$ & $18,9 \%$ & $41,2 \%$ & $20,1 \%$ & $22,3 \%$ \\
\hline ATRIA ${ }^{25}$ & $49,3 \%$ & $17,1 \%$ & $29,2 \%$ & NA & NA \\
\hline Naccarelli et al ${ }^{24}$ & $62 \%$ & $24,3 \%$ & $30,2 \%$ & NA & NA \\
\hline OFRECE ${ }^{26}$ & $76 \%$ & $24,5 \%$ & $29,4 \%$ & NA & NA \\
\hline
\end{tabular}

FA - Fibrilhação auricular; HTA - Hipertensão arterial, DM - Diabetes Mellitus, ICC - Insuficiência cardíaca congestiva, AVC - Acidente vascular cerebral, AIT - Acidente isquémico transitório, NA - Não avaliado.

do risco trombótico, de acordo com as recomendações de 2012 da ESC, ${ }^{1}$ e verificámos que apenas 2,3\% dos indivíduos com FA não valvular estudados apresentavam um risco trombótico baixo (score $=0$ ). Em contraste o estudo da Rede de Médicos Sentinela ${ }^{3}$ encontrou um valor de 32,3\% de indivíduos com baixo risco trombótico. Contudo, este valor foi encontrado com recurso a uma ferramenta de cálculo de risco diferente - a ferramenta $\mathrm{CHADS}_{2}$, em vigor na data em que o estudo foi realizado (2003) - pelo que a comparação directa destes resultados não pode ser efectuada. No entanto, esta diferença pode mostrar que um elevado número de utentes com FA que anteriormente, com recurso à ferramenta $\mathrm{CHADS}_{2}$, eram considerados de baixo risco para eventos trombóticos, deixaram de o ser com recurso à nova ferramenta $\mathrm{CHA}_{2} \mathrm{DS}_{2}$-Vasc.

No nosso estudo apenas $56,8 \%$ dos doentes estava medicado adequadamente com ACO (independentemente de estar associada ou não a terapêutica antiplaquetária), o que está de acordo com o estudo REACH ${ }^{27}$ que mostrou que cerca de $40 \%$ dos doentes diagnosticados com FA não estavam a ser tratados com terapêutica antitrombótica. Relativamente a estes resultados colocámos várias hipóteses: o recente update das normas internacionais referentes ao tratamento antitrombótico, ${ }^{1}$ que pode ainda não ter permitido a sua actualização na prática; a recusa dos doentes/cuidadores em fazer terapêutica anticoagulante quando informados dos riscos e cuidados; a dificuldade na manipulação dos anticoagulantes ao nível dos CSP ou até o excessivo receio dos eventos hemorrágicos associados a estas terapêuticas por parte dos médicos, principalmente em doentes mais idosos.

O presente estudo mostrou que $56 \%$ dos indivíduos com FA não valvular e elevado risco de eventos trombóti$\cos$ (score $\geq 1$ ) estava adequadamente sob anticoagulação oral (independentemente de estar associada ou não a terapêutica antiplaquetária), valor semelhante ao encontrado no estudo REACH ${ }^{27}$ (54\%).

Mostrou também que a utilização isolada de antiagregantes plaquetários foi observada em $29,6 \%$ dos indivíduos com elevado risco trombótico (score $\geq 1$ ), valor inferior ao estudo da Rede de Médicos Sentinela ${ }^{3}$ (48,8\%). No entanto, esta última comparação deverá ser cautelosa na medida em que os resultados foram analisados com base em ferramentas de avaliação de risco diferentes - $\mathrm{CHA}_{2} \mathrm{DS}_{2}$-Vasc no presente estudo e $\mathrm{CHADS}_{2}$ nos outros dois estudos servindo, contudo, para mostrar que existe uma elevada percentagem de indivíduos que não estão medicados de acordo com as recomendações em vigor.

Relativamente à investigação sobre a terapêutica antitrombótica, apontámos como limitação o facto de não ter sido pesquisado o motivo pelo qual o utente a estaria a fazer (ou não), de modo a perceber qual a justificação para o seu uso (ou não), o que poderá ter enviesado os resultados. Alguns utentes poderiam não estar a fazer terapêutica antitrombótica por motivo justificado ou a fazê-la por outro motivo clínico que não fosse a FA.

Neste estudo foi possível demonstrar que quanto mais elevado o risco trombótico maior a probabilidade de os doentes com FA estarem medicados com terapêutica antitrombótica, sendo, portanto, um indicador de qualidade no seguimento destes doentes, uma vez que o risco de eventos cardiovasculares (para além dos trombóticos) aumentam com o valor do score de risco, aproximando-se dos $40 \%$ em 4 anos. $^{27}$

O presente estudo utilizou uma amostra de grandes dimensões, em várias USF do concelho de Vila Nova de Gaia e utilizou na sua metodologia as mais recentes normas da ESC. Assim, reveste-se de grande utilidade para os CSP, uma vez que foram recentemente introduzidas as consultas de hipocoagulação nestas instituições, alertando os profissionais de saúde para esta problemática.

No que se refere às limitações, destaca-se a utilização de uma amostra de conveniência da população estudada e o facto do estudo se basear no registo clínico de FA (FA conhecida) em vez de se basear em novos diagnósticos, podendo, por isso, estar presente um viés de informação e de registo.

\section{CONCLUSÃO}

A prevalência global de FA conhecida encontrada neste 
estudo foi de $1,29 \%$, estando de acordo com as prevalências encontradas em estudos nacionais $(0,53 \%$ e $2,5 \%)$ e internacionais $(0,95-4,4 \%)$ e aumentando para valores superiores a $4,87 \%$ em indivíduos com idades superiores a 70 anos.

As comorbilidades mais frequentemente encontradas foram a HTA, a ICC e a DM. Pelo risco aumentado de hemorragia ou de um evento trombótico que as patologias concomitantes com a FA podem acarretar, estas devem ser adequadamente vigiadas e controladas.

Relativamente à terapêutica em curso, a maioria dos doentes encontrava-se medicada com ACO. No entanto, de acordo com as novas recomendações para o uso de terapêutica antitrombótica na FA, verificou-se que apenas $56,8 \%$ dos indivíduos estava correctamente hipocoagulada, verificando-se uma subutilização acentuada deste tratamento. Perante estes dados, torna-se premente formar os médicos relativamente às recentes actualizações no que respeita à terapêutica antitrombótica nos doentes com FA para que o risco de eventos tromboembólicos e/ou hemorrágicos seja minimizado.

Dado o provável subdiagnóstico de FA encontrado neste estudo, realça-se a importância do rastreio, nomeadamente a partir dos 65 anos de idade, de acordo com as recentes recomendações da ESC. ${ }^{1}$

\section{REFERÊNCIAS}

1. Camm AJ, Lip GY, De Caterina R, Savelieva I, Atar D, Hohnloser SH, et al. 2012 focused update of the ESC Guidelines for the management of atrial fibrillation: an update of the 2010 ESC Guidelines for the management of atrial fibrillation - developed with the special contribution of the European Heart Rhythm Association. Eur Heart J. 2012;33:2719-47.

2. Bonhorst D, Mendes M, De Sousa J, Primo J, Adragão P, Andrade S, et al. Epidemiologia da fibrilhação auricular. Rev Port Cardiol. 2010;29:1207-17

3. Ascensão P. Fibrilhação auricular e prevenção do tromboembolismo: estudo numa população de utentes de Centros de Saúde. Rev Port Clin Geral. 2006;22:13-24.

4. Bonhorst D, Mendes M, Adragão P, De Sousa J, Primo J, Leiria E, et al. Prevalence of atrial fibrillation in the Portuguese population aged 40 and over: the FAMA study. Rev Port Cardiol. 2010;29:331-50.

5. Camm AJ, Kirchhof P, Lip GYH, Schotten U, Savelieva I, Ernest S, et al. Guidelines for the management of atrial fibrillation: The Task Force for the Management of Atrial Fibrillation of the European Society of Cardiology - endorsed by the European Association for Cardio-Thoracic Surgery. Eur Heart J. 2010;31:2369-429.

6. Lloyd-Jones DM, Wang TJ, Leip EP, Larson MG, Levy D, Vasan RS, et al. Lifetime risk for development of atrial fi brillation: the Framingham Heart Study. Circulation. 2004;110:1042-6.

7. Aguiar C, De Macedo ME, De Sousa J, Ferro J, Henriques IL, Rodrigues $\mathrm{V}$, et al. Terapêutica antitrombótica da fibrilhação auricular [e-book]. Coordenação Nacional para as Doenças Cardiovasculares. Alto Comissariado da Saúde; 2009 [consultado em 2013 Jan 30]. Disponível em: http://www.portaldasaude.pt/NR/rdonlyres/6B7EB2F9-CF0B-4368A073-01C0D72998E1/0/TerapeuticaAntitromboticaFibrilhacaoAuricular. pdf.

8. Ringborg A, Nieuwlaat R, Lindgren P, Jönsson B, Fidan D, Maggioni AP, et al. Costs of atrial fibrillation in five European countries: results from the Euro Heart Survey on atrial fibrillation. Europace. 2008;10:403-11.

9. Kim MH, Johnston SS, Chu BC, Dalal MR, Schulman KL. Estimation of total incremental health care costs in patients with atrial fibrillation in the United States. Circ Cardiovasc Qual Outcomes. 2011;4: 313-20.

\section{AGRADECIMENTOS}

Os autores gostariam de agradecer aos colegas Internos de Medicina Geral e Familiar, Ana Rita Maia e Maria José Oliveira (USF São Miguel), Ana Sofia Nogueira e Rui Costa (USF São Félix da Marinha), Célia Oliva (USF Além D'Ouro), Joana Santos e João Fernandes (USF Arco do Prado), Mariana Fidalgo Leite (USF Saúde no Futuro), Sílvia Sacramento (USF Canelas), pela sua disponibilidade e colaboração na colheita dos dados para o presente estudo nas suas Unidades de Saúde Familiar.

Os autores gostariam também de agradecer a Luísa Sá, Assistente de Medicina Geral e Familiar na USF Nova Via, por toda a ajuda disponibilizada na revisão do presente estudo.

\section{OBSERVAÇÕES}

O presente estudo foi apresentado sob a forma de poster no "Update em Medicina 2014" que decorreu em Albufeira de 1 a 4 de Maio de 2014, tendo sido premiado.

\section{CONFLITOS DE INTERESSE}

Os autores declararam a inexistência de quaisquer conflitos de interesse em relação ao presente trabalho.

\section{FONTES DE FINANCIAMENTO}

O presente estudo não contou com nenhum subsídio ou bolsa para a sua realização.

10. Stewart S, Murphy N, Walker A, McGuire A, McMurray JJ. Cost of an emerging epidemic: an economic analysis of atrial fi brillation in the UK. Heart. 2004:90:286-92

11. Wolf PA, Abbott RD, Kannel WB. Atrial fibrillation; a major contributor to stroke in the elderly. The Framingham study. Arch Intern Med. 1987;147:1561-4

12. Hannon N, Sheehan O, Kelly L, Marnane M, Merwick A, Moore A, et al. Stroke associated with atrial fibrillation - incidence and early outcomes in the North Dublin Population Stroke Study. Cerebrovasc Dis. 2010;29:43-9.

13. Benjamin EJ, Levy D, Varizi SM, D'Agostino RB, Belanger AJ, Wolf PA Independent risk factors for atrial fibrillation in a population-based cohort: The Framingham Heart Study. JAMA. 1994;271:840-4.

14. Wolf PA, Abbott RD, Kannel WB. Atrial fibrillation as an independent risk factor for stroke: the Framingham Study. Stroke. 1991;22:983-8.

15. Hart RG, Pearce LA, Aguilar MI. Meta-analysis: antithrombotic therapy to prevent stroke in patients who have nonvalvular atrial fibrillation. Ann Intern Med. 2007;146:857-67.

16. You JJ, Singer DE, Howard PA, Lane DA, Eckman MH, Fang MC, et al. American College of Chest Physicians Evidence-Based Clinical Practice Guidelines: Antithrombotic therapy for atrial fibrillation. Antithrombotic therapy and prevention of thrombosis. Chest. 2012;141:e531S-75.

17. Connolly SJ, Pogue J, Hart RG, Hohnloser SH, Pfeffer M, Chrolavicius $S$, et al. Effect of clopidogrel added to aspirin in patients with atrial fibrillation. N Engl J Med. 2009;360:2066-78.

18. Connolly SJ, Ezekowitz MD, Yusuf S, Eikelboom J, Oldgren J, Parekh A et al. Dabigatran versus warfarin in patients with atrial fibrillation. $\mathrm{N}$ Eng J Med. 2009;361:1139-51.

19. Patel MR, Mahaffey KW, Garg J, Pan G, Singer DE, Hacke W, et al Rivaroxaban versus warfarin in nonvalvular atrial fibrillation. $N$ Engl $J$ Med. 2011;365:883-91.

20. Fang MC, Go AS, Chang Y, Hylek EM, Henault LE, Jensvold NG, et al. Death and disability from warfarin-associated intracranial and extracranial hemorrhages. Am J Med. 2007;120:700-5.

21. Aguiar C. Prevenção do tromboembolismo na fibrilhação auricular. Rev 
Port Cardiol. 2012;31:S17-26

22. Perfil Local de Saúde 2012: ACeS Gaia e Espinho/Gaia [homepage na Internet]. ARS Norte, IP. [Consultado em 2013 Jan 30]. Disponível em: http://portal.arsnorte.min-saude.pt/ARSNorte/dsp/ACES/ PLS2012_1914_GaiaEspinho.pdf.

23. Comissão Internacional de Classificações da WONCA. ICPC-2: Classificação Internacional de Cuidados de Saúde Primários - segunda edição. ACSS-APMCG 2011.

24. Naccarelli GV, Varker H, Lin J, Schulman KL. Increasing prevalence of atrial fibrillation and flutter in the United States. Am J Cardiol. 2009;104:1534-9.
25. Go AS, Hylek EM, Philips KA, Chang YC, Henault LE, Selby JV, et al. Prevalence of diagnosed atrial fibrillation in adults. National Implications for rhythm management and stroke prevention: the AnTicoagulation and Risk Factors in Atrial Fibrillation (ATRIA) Study. JAMA. 2001;285:23705.

26. Gomez-Doblas JJ, Muñiz J, Martin JJ, Rodríguez-Roca G, Lobos JM, Awamleh $P$, et al. Prevalencia de fibrilación auricular en España. Resultados del estudio OFRECE. Rev Esp Cardiol. 2014;67:259-69.

27. Ruff CT, Bhatt DL, Steg G, Gersh BJ, Alberts MJ, Hoffman EB, et al. Long-term cardiovascular outcomes in patients with atrial fibrillation and atherothrombosis in the REACH Registry. Int J Cardiol. 2014;170:413-8. 
Eva GOMES, Rui CAMPOS, Renata MORAIS, Marta FERNANDES

\section{Estudo FATA: Prevalência de Fibrilhação Auricular e Terapêutica Antitrombótica nos Cuidados de Saúde Primários de um Concelho do Norte de Portugal}

Acta Med Port 2015:28:35-43

Publicado pela Acta Médica Portuguesa, a Revista Científica da Ordem dos Médicos

Av. Almirante Gago Coutinho, 151

1749-084 Lisboa, Portugal.

Tel: +351218428 215

E-mail: submissao@actamedicaportuguesa.com

www.actamedicaportuguesa.com

ISSN:0870-399X | e-ISSN: 1646-0758

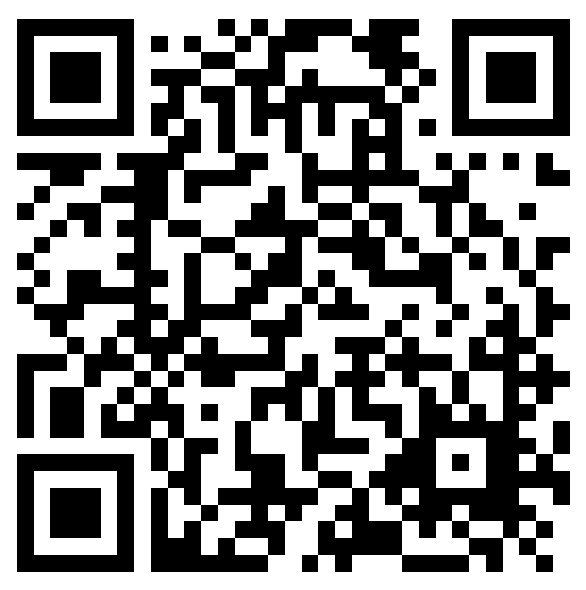

